\title{
Prevalence of deficient retro-aortic rim and its effects on outcomes in device closure of atrial septal defects
}

\author{
Michael L O'Byrne, MD, Andrew C Glatz, MD, MSCE, Sherzana Sunderji, MD, Aswathi E \\ Mathew, BS, MS, David J Goldberg, MD, Yoav Dori, MD, PhD, Jonathan J Rome, MD, and \\ Matthew J Gillespie, MD \\ Division of Cardiology, The Children's Hospital of Philadelphia and Department of Pediatrics, \\ Perelman School of Medicine at the University of Pennsylvania, Philadelphia PA
}

\begin{abstract}
Background-Deficient retro-aortic rim is of concern as a risk factor for aortic erosion following device closure of atrial septal defects (ASD). However, its prevalence and contribution to technical failure and adverse outcomes has not been delineated.

Methods-A single-center retrospective cohort study of children and adults undergoing cardiac catheterization for device occlusion of ASD from 1/1/1999 to 4/1/2012 was performed. Risk factors for technical failure and early adverse outcome were assessed using multivariate logistic regression.
\end{abstract}

Results-Four-hundred forty-five consecutive subjects of median age 5.9 years (range: 0.8-80 years) underwent catheterization during the study period. Of subjects with reviewable echocardiograms, $60 \%$ had deficient retro-aortic rim. No attempt was made at device closure in $3.6 \%$ of subjects. Of the remaining 429 subjects, $96 \%$ underwent successful device occlusion. Major early adverse events occurred in 1.2\% (95\% CI: 0.4-2.7\%) of cases, all either device embolization or malposition. Deficient retro-aortic rim was not a risk factor for composite outcome of technical failure or early major adverse event. No deaths, late re-interventions, or erosion events occurred over 2,395 total person-years of follow-up (median 5.8 years). Deficient retro-aortic rim was associated with increased risk of device impingement on the aorta, but no association was seen between device impingement or deficient retro-aortic rim and the development of new/progressive aortic insufficiency.

Conclusion-Deficient retro-aortic rim is highly prevalent but did not increase risk of adverse outcomes. Its contribution to the risk of aortic erosion could not be addressed by this study.

\section{Keywords}

septal occluder device; PEDS-pediatric interventions; heart defects; congenital; cardiac catheterization

\footnotetext{
Corresponding Author: Michael L O'Byrne, obyrnem@email.chop.edu, Division of Cardiology, The Children's Hospital of Philadelphia, $34^{\text {th }}$ St and Civic Center Blvd, Philadelphia, PA 19147, Phone: (215) 590-1790, Fax: (215) 590-5825.

The content is solely the responsibility of the authors and does not necessarily reflect the official view of the National Institutes of Health or other funding groups.
} 


\section{Introduction}

Catheter-based closure of atrial septal defects (ASD) was first reported by King and Mills in 1976[1]. Since then, device occlusion with the Amplatzer septal occluder (ASO) has been widely adopted with excellent rates of technical success[2-5], along with favorable mortality rates [6-8] and reduced overall incidence of procedural adverse events[8] relative to operative closure. Recent studies incorporating device closure with the Helex septal occluder (HSO) have shown similarly excellent outcomes[9-12]. Transcatheter device occlusion of ASD is not without risk, however, with adverse events including residual shunt[13], device embolization [3, 7, 8], pericardial effusion[6-8, 14], aortic erosion or aortic-atrial fistula[7, 8, 14], device thrombus [7, 13], endocarditis[7], dysrhythmia[7, 8], and aortic insufficiency[15] reported. Device erosion, a potentially catastrophic complication of the procedure, has received increased attention of late. A retrospective review of cases of erosion implicated deficient retro-aortic rim as a potential risk factor[14, 16], leading to changes in recommendations for device use[17-19]. However, the prevalence of deficient retro-aortic rim in children and adults who have undergone device closure has not been well defined, limiting the ability to determine to what degree it represents a true risk factor for erosion.

We performed a single-institution retrospective cohort study of children and adults undergoing catheterization for possible device occlusion of ASD to determine the prevalence of deficient retro-aortic rim and determine if it affects the risk of both technical failure and treatment-associated adverse events.

\section{Methods}

\section{Study Population}

The study protocol was approved by the institutional review board of The Children's Hospital of Philadelphia, and waiver of consent was granted. We conducted a retrospective cohort study, based on review of medical records and imaging data from subjects treated at The Children's Hospital of Philadelphia (Philadelphia, Pennsylvania). Subjects were identified through our center's cardiac catheterization laboratory and cardiac surgical databases. Children and adults across all ages were included if they underwent cardiac catheterization for possible device closure of ASD between January 1, 1999 and April 1, 2012. Subjects who were referred for operative closure without cardiac catheterization were not included. No additional exclusion criteria were applied.

\section{Study Procedures}

Medical records were reviewed including pre-catheterization assessments, inpatient admissions and emergency room visits, clinic letters, catheterization reports, angiograms, and echocardiograms performed during the study period. Subject demographics (age, sex, and race), growth parameters (height, weight, body surface area, and body mass index), and clinical history (cardiac and non-cardiac medical and operative history) were extracted. Data from cardiac catheterizations including oximetry, hemodynamics, exposure to radiation, contrast dose, and procedural adverse events were extracted from catheterization reports and 
records from hospitalization. Over the study period, the approach to device closure was consistent with device placement guided by fluoroscopy and either trans-esophageal or intracardiac echocardiography. In subjects with deficient retro-aortic rim, operators did not systematically vary their device deployment technique using previously variations[20, 21]. Specific variations in deployment technique were not recorded as part of the data collected for this study. Defect size was measured by echocardiography with balloon sizing using stop-flow technique.

Echocardiograms available on digital imaging system were reviewed by one member of the study staff (MLO). The echocardiograms reviewed included the first trans-thoracic echocardiogram prior to cardiac catheterization, the echocardiogram performed during catheterization, the first post-procedural echocardiogram, and the most recent echocardiogram within the study period. All measurements were performed blinded to original clinical reports, prior and subsequent echocardiograms, and clinical outcomes.

On pre-procedural echocardiogram septal length and defect size in orthogonal views (subcostal frontal and sagittal), tissue rims (right upper pulmonary vein, superior vena cava, inferior vena cava, mitral valve, and aorta), presence of valvar insufficiency, and the presence of right-sided atrial and ventricular enlargement were measured. On procedural echocardiograms, septal length, retro-aortic rim, and maximal defect size were measured. Deficient retro-aortic rim was defined as a retro-aortic rim measuring less than or equal to 5 $\mathrm{mm}$ on any view on the echocardiogram as has been described previously[14, 16, 22]. Defect size during balloon inflation using stop-flow technique (which was used for all cases) was also measured, with device sizing based on this measurement. Presence of residual shunt, pericardial effusion, impingement on mitral and tricuspid valve, pulmonary veins, inferior vena cava, and superior vena cava as well as impingement on or contact with the aorta, as well as the presence of aortic insufficiency, device thrombus, device malposition, arrhythmia, and device fracture were extracted from first and most recent post-procedural echocardiograms. The presence of impingement on the aorta was recorded. The manner in which the implanted device impinged on the aortic valve was also recorded, with impingement divided between "splaying" of the device around the aorta and impingement of the device on the aorta perpendicular to the aortic wall ("poking") with or without deformation of the valve.

To assess inter-rater reliability, 40 randomly selected pre-procedural studies were remeasured by another member of the study staff (DJG), representing a $20 \%$ sample of the 205 digitally available echocardiograms from subjects with single ostium secundum ASD. Intra-class correlation coefficients for continuous variables were calculated. Inter-rater reliability of all outcome measures was at least moderate for all measurements (Supplementary Table 1).

Subject medical records following catheterization were reviewed to assess for postprocedural adverse events and other clinical outcomes. Subjects were considered at risk if an attempt was made to deploy (though not necessarily release) a device. Technical success was defined as a successful deployment of a device without residual shunt greater than 3 $\mathrm{mm}$. Major adverse events included device embolization, malposition followed by repeat 
intervention, procedure-associated cerebrovascular events, heart block persisting after the procedure, and death. Minor adverse events noted included arrhythmia, fever, hematoma (defined by follow-up notes from cardiologists or visits to other healthcare facilities), emesis, and pericardial effusion (not requiring intervention).

Complications were sub-divided between early (less than 30 days of initial procedure) and late (greater than 30 days). Early follow-up time of included subjects was uniform, so event rates were described as risk per case. Late follow-up was not uniform, so duration of followup was measured in terms of events per person-years of follow-up. Clinical follow-up was calculated in two ways: first as time from ASD device deployment until end of study period and second as time elapsed between initial catheterization until most recent documented clinical visit at our center. This provides bounds for measurement of time at risk. Echocardiographic follow-up time was determined as the time elapsed from catheterization to most recent echocardiogram in the study period.

\section{Statistical Analysis}

Standard descriptive statistics were calculated. Continuous variables are expressed as mean \pm standard deviation or median (range and interquartile range (IQR)) as appropriate. Categorical variables are described as proportions and counts.

The primary outcome for early adverse events was a composite of technical failure and/or early, major adverse event as defined above. Assessment of risk factors for primary composite outcome was performed using logistic regression. Because this was an exploratory analysis, bi-variable screening was used to identify risk factors, with potential risk factors included in the initial model if $p<0.2[23,24]$. Prior to any testing, the decision was made to include deficient retro-aortic rim in the final model as the primary exposure. Backwards selection was used to refine the model with variables retained if $p<0.05$ or if their inclusion resulted in a change in the odds ratio of $>20 \%$. Model fit was assessed post hoc with likelihood ratio test for nested models or comparison of Akaike information criterion for non-nested models[24]. Since the cohort size was fixed, no formal procedures were performed to calculate statistical power. No formal compensation for multiple comparisons was performed. For subjects with missing data, case elimination was used, and no attempt was made to replace missing data via imputation. A threshold for statistical significance was set at $p<0.05$. All data analysis was performed using Stata SE v12 (Statacorp, College Station, TX).

\section{Results}

\section{Study population}

Between January 1, 1999 and April 1, 2012, 445 consecutive children and adults underwent cardiac catheterization with an indication for possible device closure of an ASD (Figure 1). Their median age and weight were 5.9 years (range: 0.8-80 years, IQR: 3.6-14.2 years) and $21.7 \mathrm{~kg}$ (range $5.4-132.5 \mathrm{~kg}$, IQR: $15.2-15.7 \mathrm{~kg}$ ) respectively (Table 1). Fifteen percent of subjects had congenital heart disease in addition to their ASD, which includes 34 subjects who had residual ASD following other cardiac operations (8\% of the study cohort) and 32 
subjects ( $7 \%$ of the cohort) who had other anatomic cardiac disease without previous operation. These counts do not include subjects with mild valvar pulmonic stenosis.

Of the original 445 subjects, 248 (56\%) had reviewable pre-procedural echocardiograms (Figure 1). Of the 330 subjects with single ostium secundum ASD, 205 (62\%) had reviewable pre-procedural TTE (Table 2). Amongst subjects with isolated secundum ASD, the median size of secundum ASD on pre-procedural transthoracic echocardiograms was 9.8 $\mathrm{mm}$ (range: 1-20.5 mm IQR: 7.5-12.2 mm) in subcostal frontal view and $9.5 \mathrm{~mm}$ (range: 3.9-26.3 mm IQR: 7.7-12.1 mm) in the subcostal sagittal view (Table 2). The median defect size with balloon sizing using stop-flow technique was $14 \mathrm{~mm}$ (range 5-38 mm IQR: 12-19 $\mathrm{mm}$ ). The median retro-aortic tissue rim was $4.2 \mathrm{~mm}$ (range 0-15.3 mm IQR: 2.9-6.2), with $60 \%$ of subjects having deficient retro-aortic rim. The prevalence of deficient retro-aortic rim was $61 \%(116 / 190)$ in subjects with age $<18$ years with images of the retro-aortic rim unavailable in six subjects, and 44\% (4/9) in subjects with age $>18$ years, but this difference was not significant $(p=0.3)$

Of the original 445 subjects, $3.6 \%(n=16)$ were deemed inappropriate for device closure and were referred for operative closure without attempted device placement. These were secondary to a combination of defect size, position, and rim size. None were due to insufficient retro-aortic rim. Of the remaining 429 subjects who underwent attempted device closure, $96 \%(n=411)$ were technically successful. A single ASO device was used in $61 \%$ of cases; a single HSO device was used in $24 \%$ of cases; and an NMT Starflex device was used in 9\%. Results of subjects in whom multiple devices were used are summarized in Table 3. For subjects with single secundum ASD and deficient retro-aortic rim $(\mathrm{n}=116)$, device closure was accomplished with ASO in $67 \%$ of subjects and HSO in $33 \%$ of subjects. No other devices were used for this subgroup.

In subjects who underwent device occlusion with ASO device, the median ratio of device diameter to balloon-sized defect diameter was 1.0 (range: 0.8-1.8, IQR: 1.0-1.1) (Table 3). Of subjects with single secundum ASD occluded with ASO $(n=217)$, only one subject $(0.5 \%$ 95\% CI: $0.01-2.5 \%$ ) had an ASO diameter to defect ratio $>1.5: 1$. For subjects with single secundum ASD occluded with HSO device $(\mathrm{n}=81)$, the median ratio of HSO diameter to defect size was 2.1 (range: $1.5-3.6$, IQR: 1.7-3.1).

\section{Follow-up and Adverse Events}

The 413 subjects who underwent device closure had median total follow-up of 5.8 years (range: 12 days to 12.6 years) for total time at risk of 2395 person-years. In $83 \%$ of subjects, a follow-up visit at our center was documented after discharge with most recent visit at median time 2.2 years (range 2 days to 12.5 years) representing 1079 person-years, of time at risk. Follow-up echocardiograms could be reviewed in 330 subjects (80\%). Median echocardiographic follow-up was 1.5 years (range: 1 day to 12.5 years) for a total of 845 person-years, of which 267 subjects $(65 \%)$ had echocardiograms after the first postprocedure day.

Of the 413 subjects with successful occlusion of their ASD, 319 subjects (77\%) had clinical follow-up of at least 30 days. Adverse events within the first 30 days of follow up are 
summarized in Table 4. Major adverse events occurred in $1.1 \%$ of subjects (95\% CI: $0.4-$ $2.6 \%$ ), including 3 subjects ( $0.7 \%$ of those undergoing device closure, $95 \%$ CI: $0.1-2.0 \%)$ in whom device embolization occurred, and 2 subjects $(0.4 \%$ of those undergoing device closure, $95 \% 0.1-1.6 \%$ ) in whom device malposition resulted in re-intervention. All reinterventions were accomplished through repeat catheterization. In these re-interventions the only adverse event was new mild mitral regurgitation in a single patient after an HSO device embolized to the LV and was retrieved with snare catheter. One subject presented to the emergency department with fever and emesis two days after catheterization with a small pericardial effusion and was admitted for observation. The patient was eventually discharged without receiving medical therapy or any procedure to drain the effusion. Eight other subjects had trivial pericardial effusions (total $n=9,5.7 \%$ of subjects who underwent device closure with complete 30 day follow-up, 95\% CI: 3.2-9.4\%), none of which resulted in emergency room visits or hospitalization and all of which were self-resolved.

A total of 15 subjects ( $4.6 \% 95 \%$ CI: $2.6-7.5 \%)$, presented to an emergency room within 30 days of their procedure, with 3 resultant hospital admissions (0.9\% 95\% CI: $0.2-2.7 \%)$. In addition to the previously mentioned subject with a small pericardial effusion, 7 subjects presented with headaches, 3 of whom were admitted for observation and symptom management. Two subjects with acute chest pain were seen in emergency departments with one admitted for observation; evaluation demonstrated no evidence of a cardiac etiology. One subject with a hematoma at access site in the groin was seen and discharged from the emergency department without additional intervention. The remaining four visits were for chief complaints (gastroenteritis, viral respiratory infection, lymphadenitis, and chronic constipation) unrelated to catheterization procedure.

Risk factors for the composite outcome (technical failure to close the defect with transcatheter device or major adverse event within 30 days of catheterization) were assessed using bi-variable logistic regression, the results of which are summarized in Table 5. In multivariable regression, the presence of deficient retro-aortic rim did not increase the risk of the composite outcome, and if anything appeared to have a non-significant association with reduced risk of combined adverse events (OR: 0.28 95\% CI: 0.08-1.01 p=0.05). Ratio of pulmonary to systemic blood flow was associated with increased risk of composite outcome (OR 2.57 95\% CI: 1.2-5.4, $p=0.013$ ).

After thirty days, no device embolization, malposition, device thrombus, new arrhythmia, stroke, or death were seen. No aortic erosions were seen, and no new pericardial effusions were noted. Of the trivial residual shunts, none underwent further intervention. New or increased aortic insufficiency was seen in 7 subjects (2\% 95\% CI: $0.9-4.5 \%$ ) between their pre-catheterization echocardiogram and most recent post-catheterization echocardiogram. One subject developed new mild aortic insufficiency, with the remainder developing new trivial aortic insufficiency. The association between deficient retro-aortic rim, device impingement, and aortic insufficiency were examined. In the 181 subjects with isolated secundum ASD and complete data, deficient retro-aortic rim was associated with increased risk of impingement of the device on the aortic valve (relative risk (RR): $2.195 \%$ CI: $1.5-$ $3.0, \mathrm{p}<0.0001$ ) (Table 6). There was a significant association between deficient retro-aortic rim and the two types of device impingement, with increased risk of device impingement 
without splaying ("poking") (RR: 1.6) and impinging with splaying of the device (RR: 2.2) in subjects with deficient retro-aortic rim $(p=0.002)$. Only a single example of poking with deformation of the valve was seen, so statistical inference was impossible. The incidence of new or increased aortic insufficiency was not associated with impingement of ASD device $(p=0.66)$ nor with the specific type of impingement $(p=0.85)$ or deficient retro-aortic rim $(p=0.91)$. The low event rate was prohibitive for multivariate analysis. Other risk factors for development of aortic insufficiency were explored using additional univariate analyses. Device choice between ASO and HSO was not associated with risk of new aortic insufficiency ( $\mathrm{p}=0.96$ ). Additionally, no association was seen between the ratio of ASO size to septum ( $p=1.0)$, HSO size to septum ( $p=0.67)$, and ASO left atrial disc to septum $(p=0.92)$.

\section{Discussion}

This cohort study summarizes the experience with device occlusion of ASD at a single center over a twelve-year period. In this cohort, deficient retro-aortic rim was highly prevalent. The clinical significance of deficient retro-aortic rim is not clear. Deficient retroaortic rim was associated with an increased risk of impingement of the device on the aorta but was not associated with increased risk of early adverse outcome or development of aortic insufficiency. There were no aortic erosions, and there were no late, major adverse outcomes. Thus, we cannot comment on the contribution of deficient retro-aortic rim to the risk of late adverse events or aortic erosion. We can state that it was not an independent risk factor for early adverse events or technical failure.

Device occlusion of ASD in the modern era has demonstrated excellent efficacy with procedural morbidity and mortality which compare favorably to operative closure of ASD[2-5, 7-12, 25]. However, the occurrence of aortic erosion, a rare but potentially catastrophic complication has inspired debate regarding the safety of device occlusion of ASD. A review of known cases of aortic erosion implicated deficient retro-aortic rim (along with device-to-defect oversizing) as a potential risk factor for aortic erosion[14]. It is difficult to assess the contribution of deficient retro-aortic rim to risk, in part because its prevalence in patients referred for device closure has not been well described. Detailed echocardiographic assessment has not been included, to our knowledge, in studies of device occlusion. One study by Butera and colleagues focusing on adult patients (median age: 29 years), measured the prevalence of deficient retro-aortic rim of 24\%[26]. Petit and colleagues, in a series of young children ( $<3$ years of age) measured a prevalence of 59\%[12], which is identical to that measured in this study (59\% with 95\% CI: 53-67\%). It should be highlighted that the current study includes subjects from infancy to adulthood. No significant difference was demonstrated in the prevalence of deficient retro-aortic rim is highly prevalent between children and adults, but the small number of adults in our series limited the statistical power. In this study, an attempt was made to determine if deficient retro-aortic rim was a risk factor for adverse outcomes across longitudinal follow-up and, failing that, if there was evidence that it increased risk of precursors to aortic erosion.

Deficient retro-aortic rim did not represent a risk factor for our composite outcome of technical failure or early major adverse events. Though not significant, the point estimate of 
its association with technical failure or major adverse outcome suggested that it was a protective factor. There is no mechanism that suggests why this would be true. It is unlikely the result of selection bias (e.g. excluding higher risk patients with deficient retro-aortic rim), since the study includes periods before and after deficient retro-aortic rim was considered a risk factor for bad outcome. The magnitude of left to right shunt (ratio of pulmonary to systemic blood flow) was an independent risk factor for our composite outcome. The magnitude of shunt was correlated with defect size (both on pre-procedural and procedural echocardiograms (data not shown), and though the analysis was not designed to assess for it, it appears that it is a risk factor for increase risk of technical failure or major adverse outcome.

No aortic erosions (or other late adverse outcomes) occurred in our series, so the association between erosion and deficient retro-aortic rim cannot be assessed from our data. Initial reports proposed the risk of aortic erosion was 1 in 1000 cases[14], with estimates of risk in the United States of $0.07-0.11 \%$ [27]. In this cohort, ASO devices were placed in 271 subjects (250 received single devices, 21 received either multiple ASO or ASO and another device to occlude multiple ASD), illustrating the limitations of even relatively large single center studies to address this issue.

However, utilizing the available serial echocardiograms in our series, we attempted to assess potential risk factors for adverse outcomes, including device impingement on the aorta and new or progressive aortic insufficiency. Impingement has not been shown to be a sufficient or necessary cause for aortic erosion, but insofar as it indicates contact between the device and the aorta it was felt to be a potential risk factor. Development or progression of aortic insufficiency potentially indicates disruption of normal aortic valve function with ASD device present. It has been noted following device occlusion of ASD in adults[15] but not associated with increased risk of any specific clinical outcomes (mortality or specific morbidity). The risk of new/progressive aortic insufficiency in our cohort was much lower than that reported in a single study of adults who underwent device occlusion, and the severity of resultant aortic insufficiency was qualitatively less. From the current study, we can conclude that impingement occurs with and without deficient retro-aortic rim but more frequently with deficient retro-aortic rim. Aortic insufficiency developed in subjects without clear association to deficient retro-aortic rim or to device impingement. Further study is necessary to determine if impingement contributes to the risk of erosion, and whether this is a potentially modifiable risk factor. What is clear is that the causes of impingement and progressive insufficiency may be complicated and that single anatomic measurements may be insufficient to predict risk.

\section{Limitations of the study}

No late adverse events occurred, so, as noted, it has limited utility in determining the association between deficient retro-aortic rim and these late outcomes. In addition, the results are derived from a retrospective study at a single center and reflect practice patterns at our institution. This limits our ability to comment on risk factors that are not observed commonly. For example, Amin and colleagues identified device over-sizing (ratio of ASO diameter to diameter of defect on balloon sizing $>1.5: 1$ ) as a potential risk factor for aortic 
erosion[14]. Only one subject in our cohort had an ASO to defect ratio >1.5:1. Another limitation of this study is the presence of missing data. Echocardiograms were reviewable in $56 \%$ of subjects. Subjects with echocardiograms that were not reviewable had procedures in an earlier period (preceding the adoption of digital recording and storage of studies at our institution). It is unlikely that the prevalence of deficient retro-aortic rim changed over the study period, and in an earlier era where deficient rim was not of clinical concern, prevalence in treated subjects may have been even higher. Follow-up was also not uniform; $77 \%$ of subjects received outpatient follow-up within our clinic system. Subjects without early clinical follow-up were not considered in calculations of risk for early adverse events. To measure the time at risk for late adverse events in the cohort, both a total follow-up time and a time from procedure to most recent clinic visit are included to provide conservative and liberal bounds. For other analyses, subjects with missing data were eliminated from analysis by case deletion. Finally, the low incidence of adverse outcomes introduces the potential for type II error. Where possible, both $p$-values and confidence intervals have been included to accurately reflect the magnitude of uncertainty in risk. Further studies from multiple centers especially registry data would be valuable to overcome these limitations and improve the generalizability of this study.

\section{Conclusion}

Deficient retro-aortic rim is highly prevalent in subjects referred for device occlusion of ASD and to the best of our knowledge does not represent a risk factor for technical failure, early adverse events, or development of aortic insufficiency.

\section{Supplementary Material}

Refer to Web version on PubMed Central for supplementary material.

\section{Acknowledgments}

Dr. O'Byrne is supported by grants from the National Institute of Health (T32HL007915) and is the recipient of the Entelligence Young Investigator Grant. Dr. Gillespie is supported by grants from the AHA and NIH, along with research support from Medtronic. Dr. Glatz is supported by grants from the AHA and Children's Heart Foundation. Dr. Goldberg is supported by the National Institute of Health (NR002093 and U10HL068270). Dr. Dori receives research support from Siemens. The authors have no other disclosures.

\section{Abbreviations}

$\begin{array}{ll}\text { ASD } & \text { atrial septal defect } \\ \text { ASO } & \text { Amplatzer septal occluder } \\ \text { HSO } & \text { Helex septal occluder } \\ \text { IQR } & \text { Inter-quartile range } \\ \text { CI } & \text { confidence interval } \\ \text { OR } & \text { odds ratio } \\ \text { Qp:Qs } & \text { ratio of pulmonary to systemic blood flow }\end{array}$




\section{References}

1. King TD, Thompson SL, Steiner C, Mills NL. Secundum atrial septal defect. Nonoperative closure during cardiac catheterization. JAMA: The Journal of the American Medical Association. 1976; 235:2506-2509. [PubMed: 946659]

2. Du ZD, Hijazi ZM, Kleinman CS, et al. Comparison between transcatheter and surgical closure of secundum atrial septal defect in children and adults: results of a multicenter nonrandomized trial. JAC. 2002; 39:1836-1844.

3. Levi DS, Moore JW. Embolization and retrieval of the Amplatzer septal occluder. Cathet Cardiovasc Intervent. 2004; 61:543-547.10.1002/ccd.20011

4. Chessa M, Carminati M, Butera G, et al. Early and late complications associated with transcatheter occlusion of secundum atrial septal defect. JAC. 2002; 39:1061-1065.

5. Wang J-K, Tsai S-K, Wu M-H, et al. Short- and intermediate-term results of transcatheter closure of atrial septal defect with the Amplatzer Septal Occluder. American Heart Journal. 2004; 148:511517.10.1016/j.ahj.2004.01.030 [PubMed: 15389241]

6. DiBardino DJ, McElhinney DB, Kaza AK, Mayer JE Jr. Analysis of the US Food and Drug Administration Manufacturer and User Facility Device Experience database for adverse events involving Amplatzer septal occluder devices and comparison with the Society of Thoracic Surgery congenital cardiac surgery database. The Journal of Thoracic and Cardiovascular Surgery. 2009; 137:1334-1341.10.1016/j.jtcvs.2009.02.032 [PubMed: 19464444]

7. Delaney JW, Li JS, Rhodes JF. Major Complications Associated with Transcatheter Atrial Septal Occluder Implantation: A Review of the Medical Literature and the Manufacturer and User Facility Device Experience (MAUDE) Database. Congenital heart disease. 2007; 2:1-9.

8. Butera G, Biondi-Zoccai G, Sangiorgi G, et al. Percutaneous versus surgical closure of secundum atrial septal defects: a systematic review and meta-analysis of currently available clinical evidence. EuroIntervention. 2011; 7:377-385.10.4244/EIJV7I3A63 [PubMed: 21729841]

9. Latson LA, Jones TK, Jacobson J, et al. Analysis of factors related to successful transcatheter closure of secundum atrial septal defects using the HELEX septal occluder. American Heart Journal. 2006; 151:1129.e7-1129.e11.10.1016/j.ahj.2006.01.005 [PubMed: 16644351]

10. Kutty S, Abu Hazeem A, Brown K, et al. Long-Term (5- to 20-Year) Outcomes After Transcatheter or Surgical Treatment of Hemodynamically Significant Isolated Secundum Atrial Septal Defect. AJC. 2012; 109:1348-1352.10.1016/j.amjcard.2011.12.031

11. Vincent RN, Raviele AA, Diehl HJ. Single-center experience with the HELEX septal occluder for closure of atrial septal defects in children. Journal of Interventional Cardiology. 2003; 16:79-82. [PubMed: 12664820]

12. Petit CJ, Justino H, Pignatelli RH, et al. Percutaneous Atrial Septal Defect Closure in Infants and Toddlers: Predictors of Success. Pediatr Cardiol. 2012; 34:220-225.10.1007/s00246-012-0413-6 [PubMed: 22806712]

13. Becker M, Frings D, Schröder J, et al. Impact of occluder device type on success of percutaneous closure of atrial septal defects--a medium-term follow-up study. Journal of Interventional Cardiology. 2009; 22:503-510.10.1111/j.1540-8183.2009.00507.x [PubMed: 19821880]

14. Amin Z, Hijazi ZM, Bass JL, et al. Erosion of Amplatzer septal occluder device after closure of secundum atrial septal defects: Review of registry of complications and recommendations to minimize future risk. Cathet Cardiovasc Intervent. 2004; 63:496-502.10.1002/ccd.20211

15. Schoen SP, Boscheri A, Lange SA, et al. Incidence of aortic valve regurgitation and outcome after percutaneous closure of atrial septal defects and patent foramen ovale. Heart. 2008; 94:844847.10.1136/hrt.2007.132662 [PubMed: 18070946]

16. Amin Z. Echocardiographic predictors of cardiac erosion after amplatzer septal occluder placement. Catheter Cardiovasc Interv. 201310.1002/ccd.25175

17. Mahapatra, S.; Graves, AM. Letter to Physicians. 2013. p. 1-2.professionalsjmcom 
18. Amplatzer Septal Occluder and Delivery System: Instructions for Use. 2012. p. 1-16.professionalsjmcom

19. Rare Serious Erosion Events Associated with St. Jude Amplatzer Atrial Septal Occluder (ASO). United States Food and Drug Administration; 2013.

20. Amin Z. Transcatheter closure of secundum atrial septal defects. Cathet Cardiovasc Intervent. 2006; 68:778-787.10.1002/ccd.20872

21. Abdullah Al-Qbandi, MH.; Hijazi, ZM. Device Closure of Difficult ASDs. In: Hijazi, ZM.; Feldman, T.; Abdullah Al-Qbandi, MH.; Sievert, H., editors. Transcatheter Closure of ASDs and PFOs A Comprehensive Assessment. Cardiotext; Minneapolis: 2010. p. 177-195.

22. Mallula K, Amin Z. Recent Changes in Instructions for Use for the Amplatzer Atrial Septal Defect Occluder: How to Incorporate These Changes While Using Transesophageal Echocardiography or Intracardiac Echocardiography? Pediatr Cardiol. 2012; 33:995-1000.10.1007/s00246-012-0323-7 [PubMed: 22576763]

23. Sun GW, Shook TL, Kay GL. Inappropriate use of bivariable analysis to screen risk factors for use in multivariable analysis. Journal of Clinical Epidemiology. 1996; 49:907-916. [PubMed: 8699212]

24. Vittinghoff, E.; Glidden, DV.; Shiboski, SC.; McCulloch, CE. Regression Methods in Biostatistics: Linear, Logistic, Survival, and Repeated Measures Models. 2. Springer; New York: Predictor Selection; p. 395-429.

25. DiBardino DJ, Mayer JE Jr. Continued controversy regarding adverse events after Amplatzer septal device closure: Mass hysteria or tip of the iceberg? The Journal of Thoracic and Cardiovascular Surgery. 2011; 142:222-223.10.1016/j.jtcvs.2011.04.014 [PubMed: 21683840]

26. Butera G, Romagnoli E, Carminati M, et al. Treatment of isolated secundum atrial septal defects: Impact of age and defect morphology in 1,013 consecutive patients. American Heart Journal. 2008; 156:706-712.10.1016/j.ahj.2008.06.008 [PubMed: 18926151]

27. Diab K, Kenny D, Hijazi ZM. Erosions, erosions, and erosions! Device closure of atrial septal defects: How safe is safe? Cathet Cardiovasc Intervent. 2012; 80:168-174.10.1002/ccd.24517 


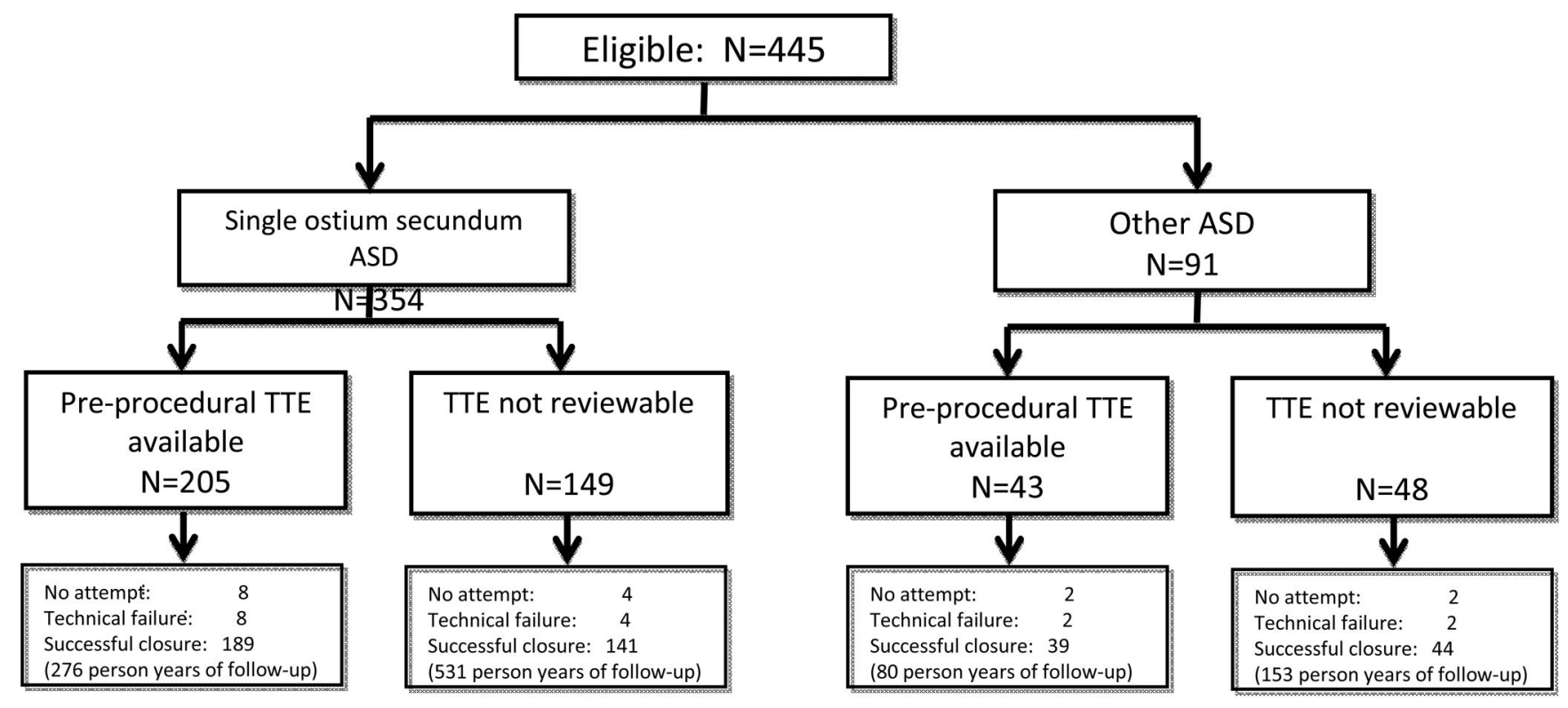

Figure 1.

Study population 


\section{Table 1}

\section{Study Population}

\begin{tabular}{|c|c|}
\hline $\mathrm{N}$ & 445 \\
\hline Age (years) & 5.9 (range: $0.8-80$, IQR: $3.6-14.2$ ) \\
\hline Male sex & $38 \%(n=171)$ \\
\hline \multicolumn{2}{|l|}{ Race } \\
\hline White & $71 \%(315)$ \\
\hline African-American & $10 \%(46)$ \\
\hline Asian, Pacific Islander, or Native Hawaiian & $3 \%(16)$ \\
\hline Native American or Native Alaskan & $0 \%(0)$ \\
\hline Other ${ }^{*}$ & $15 \%(68)$ \\
\hline Weight $(\mathrm{kg})$ & 21.7 (range: $5.4-132.5$, IQR: $15.2-52.7$ ) \\
\hline Height $(\mathrm{cm})$ & 116 (range: 54-194, IQR: 97.8-157) \\
\hline BMI $\left(\mathrm{kg} / \mathrm{m}^{2}\right)$ & 17 (range: $13-43$, IQR: $14-21)$ \\
\hline $\operatorname{BSA}\left(\mathrm{m}^{2}\right)$ & 0.8 (range: $0.4-2.6$, IQR: $0.6-1.5$ ) \\
\hline Genetic Syndrome $\%(\mathrm{n})^{\dagger}$ & $6.7 \%(30)$ \\
\hline \multicolumn{2}{|l|}{ Defect type } \\
\hline Ostium secundum defect & $79.3 \%(353)$ \\
\hline Multi-fenestrated atrial septal defect & $9.0 \%(40)$ \\
\hline Residual ASD after operation for other $C H D^{\ddagger}$ & $7.6 \%(34)$ \\
\hline Patent foramen ovale & $3.2 \%(14)$ \\
\hline Other ${ }^{\S}$ & $0.9 \%(4)$ \\
\hline \multicolumn{2}{|l|}{ Indication for procedure } \\
\hline Elevated Qp:Qs (>=1.5:1) & $72.1 \%(321)$ \\
\hline Volume overload of right ventricle and Qp:Qs $<1.5^{\mathscr{I}}$ & $17.3 \%(77)$ \\
\hline Right to left shunt & $7.0 \%(31)$ \\
\hline Transient ischemic attack/Cerebrovascular accident $\#$ & $3.1 \%(14)$ \\
\hline Other $^{* *}$ & $0.4(2)$ \\
\hline Other structural cardiac disease ${ }^{\dagger \dagger}$ & $7.5 \%(32)$ \\
\hline Previous tachydysrhythmia & $3.2 \%(14)$ \\
\hline Previous heart block $\S \S$ & $0.9 \%$ \\
\hline
\end{tabular}

Abbreviations: ASD atrial septal defect, BMI body mass index, BSA body surface area, CHD congenital heart disease, Qp:Qs ratio of pulmonary to systemic blood flow, Median (range and inter-quartile range) or \% (n)

Includes two or more races, deferred, or recorded as "other"

${ }^{\dagger}$ Trisomy $21(\mathrm{n}=10)$, chromosomal abnormality not otherwise specified ( $\left.\mathrm{n}=5\right), 22 \mathrm{q} 11.2$ deletion ( $\left.\mathrm{n}=2\right)$, Noonan's Syndrome ( $\left.\mathrm{n}=2\right)$, WolffHirschorn $(n=2)$, and mosaic trisomy 13, unbalanced translocation of chromosome, MSUD, CHAOS syndrome, Perlman Syndrome, Turner Syndrome, 4q deletion, Soto's syndrome, XXXXY (all n=1)

${ }^{*}$ Pulmonary atresia intact ventricular septum $(\mathrm{n}=12)$ tetralogy of Fallot $(\mathrm{n}=10)$, transposition of the great arteries status post arterial switch operation $(n=6)$, tetralogy of Fallot pulmonary atresia $(n=2)$ hypertrophic cardiomyopathy s/p right ventricular outflow tract patch $(n=1)$ partial atrioventricular canal status post repair $(n=1)$, ASD repair at outside hospital $(n=1)$, interrupted aortic arch $(n=1)$, surgical valvotomy of pulmonary stenosis $(\mathrm{n}=1)$. 


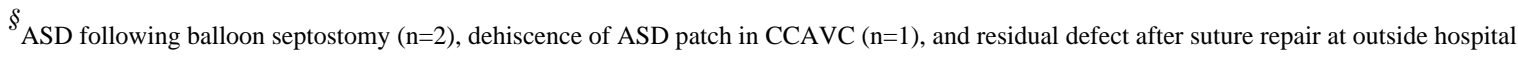
$(\mathrm{n}=1)$

${ }^{\text {II }}$ Includes 8 subjects with documented RV dilation and unretrievable Qp:Qs

\# One subject had Qp:Qs=1.5 in addition to previous CVA

*** Prophylaxis prior to transvenous device $(\mathrm{n}=1)$ and migraine headache $(\mathrm{n}=1)$

${ }^{\dagger \dagger}$ Structural heart disease $15.1 \%$ (7.2\% of which are ASD that were not left after operations) Ebsteins ( $\left.\mathrm{n}=12\right)$, PDA (n=10), restrictive VSD ( $\mathrm{n}=5$ ), double aortic arch $(n=1)$ pulmonary AVM $(n=1)$, coronary to RPA fistula $(n=1)$, valvar and subvalvar pulmonic stenosis $(n=1)$, and PDA with VSD $(\mathrm{n}=1)$.

Previous arrhythmia $4.0 \%$

${ }^{\#}$ Supraventricular tachycardia not otherwise specified $(\mathrm{n}=6)$, atrioventricular nodal reentrant tachycardia $(\mathrm{n}=4)$, long QT syndrome $(\mathrm{n}=2)$, ectopic atrial tachycardia $(\mathrm{n}=1)$, and ventricular tachycardia $(\mathrm{n}=1)$.

$\S \S$ Congenital complete heart block $(\mathrm{n}=3)$ and first degree AV conduction delay $(\mathrm{n}=1)$ 


\section{Table 2}

\section{Anatomy of defects (restricted to secundum ASD)}

\begin{tabular}{|c|c|}
\hline $\mathrm{N}$ & $205^{*}$ \\
\hline \multicolumn{2}{|l|}{ Defect Size (mm) } \\
\hline Subcostal frontal $(n=205)$ & 9.8 (range: $1.0-20.5$, IQR: 7.5-12.2) \\
\hline Subcostal sagittal $(n=202)$ & 9.5 (range: 3.9-26.3, IQR: 7.7-12.1) \\
\hline Apical 4 chamber $(n=195)$ & 10.5 (range: $3.4-30.7$, IQR: $8.3-13.1$ ) \\
\hline Defect largest diameter on TEE $(n=213)$ & 10.0 (range: $0.8-28.0$, IQR: 7.7-13.9) \\
\hline Diameter using stop-flow on catheterization report $(n=322)$ & 14 (range: 5-38, IQR: 12-19) \\
\hline Diameter using stop-flow measured on TEE $(n=198)$ & 14 (range: 4-42.6, IQR: $11.4-18.3$ ) \\
\hline \multicolumn{2}{|l|}{ Septum length (mm) } \\
\hline Subcostal frontal $(n=205)$ & 38.8 (range: $11.2-65.5$, IQR: $33.1-45.2$ ) \\
\hline Subcostal sagittal $(n=202)$ & 38.0 (range: $16.8-63.3$, IQR: $30.9-45.5$ ) \\
\hline Apical 4 chamber $(n=195)$ & 33.9 (range: $12.2-60.4$, IQR: 29.3-39.6) \\
\hline \multicolumn{2}{|l|}{ Tissue Rim (mm) } \\
\hline Right upper pulmonary vein $(\mathrm{n}=203)$ & 12.4 (range: 3-27.4, IQR: 10.1-16.0) \\
\hline Superior vena cava $(n=201)$ & 13.1 (range: 4-30.2, IQR: 10.3-16.3) \\
\hline Inferior vena cava $(n=201)$ & 15.6 (range: $6.3-44.3, \mathrm{IQR}: 13.3-19.5$ ) \\
\hline Mitral valve $(n=194)$ & 12.7 (range: 5.4-32.0, IQR: 10.1-15.2) \\
\hline Retro-aortic $(n=199)$ & 4.2 (range: $0.8-15.3$, IQR: 2.9-6.2) \\
\hline Deficient retro-aortic rim \% (n) & $60 \%(120)$ \\
\hline
\end{tabular}

* Those with digitally available procedural echocardiograms 


\section{Table 3}

Characteristics of catheterization procedures

\begin{tabular}{|c|c|}
\hline $\mathrm{N}$ & 445 \\
\hline Ruled anatomically inappropriate without device release \% (n) & $3.6 \%(16)$ \\
\hline Deployed but removed (n=429) \% (n) & $4.0 \%(18)$ \\
\hline Successful deployment $(\mathrm{n}=429) \%(\mathrm{n}){ }^{*}$ & $96 \%(411)$ \\
\hline \multicolumn{2}{|l|}{ Devices used ${ }^{\dagger}$} \\
\hline Amplatzer septal occluder & $60.5 \%(250)$ \\
\hline Helex septal occluder & $24.2 \%(100)$ \\
\hline Amplatzer multi-fenestrated septal occluder & $1.2 \%(5)$ \\
\hline Starflex device & $9.0 \%(37)$ \\
\hline Multiple devices & $5.1 \%(21)$ \\
\hline Other cardiac procedures performed & $7.2 \%(32)$ \\
\hline Other transcatheter intervention & $4.0 \%(18)$ \\
\hline Electrophysiology study & $2.2 \%(10)$ \\
\hline Coronary angiography & $0.7 \%(3)$ \\
\hline \multicolumn{2}{|l|}{ Qp:Qs } \\
\hline All subjects $(n=427)$ & 1.7 (Range: $0.4-5.2$, IQR: $1.4-2.2$ ) \\
\hline Only subjects with secundum $A S D(n=342)$ & 1.8 (Range: $0.7-5.2$, IQR: $1.4-2.3$ ) \\
\hline Ratio of ASO diameter:ASD on stop-flow $(\mathrm{n}=250)$ & 1.0 (Range $0.8-1.8$, IQR: $1.0-1.1$ ) \\
\hline Ratio of ASO LA disc:septum ( $n=155)$ & 0.7 (Range $0.4-1.3$, IQR: $0.5-1.0$ ) \\
\hline Ratio of ASO RA disc:septum ( $\mathrm{n}=155)$ & 0.6 (Range: $0.3-1.1$, IQR: $0.4-0.9$ ) \\
\hline Ratio of HELEX diameter: ASD on stop-flow $(\mathrm{n}=90)$ & 2.1 (Range: $1.5-3.6, \mathrm{IQR}: 1.7-3.6$ ) \\
\hline Ratio of HELEX diameter:septum ( $\mathrm{n}=65$ ) & 0.6 (Range: $0.3-1.0,0.4-1.0$ ) \\
\hline \multicolumn{2}{|l|}{ Radiation exposure } \\
\hline Fluoroscopy Time (minutes) $(\mathrm{n}=359)$ & 17.1 (range: $1.0-113.9$, IQR: $12.1-27.8$ ) \\
\hline Total Skin Dose A (milliGray) $(\mathrm{n}=110)$ & 85.5 (range: $8.1-2334$, IQR: 52.8-228) \\
\hline Total Skin Dose B (milliGray) $(\mathrm{n}=110)$ & 61.4 (range: $4.3-3358$, IQR: 26.3-145) \\
\hline Dose Area Product A (microGray $\left./ \mathrm{m}^{2}\right)(\mathrm{n}=107)$ & 756.2 (range: 70.6-22838, IQR: 387.6-1796.7) \\
\hline Dose Area Product B (microGray $\left./ \mathrm{m}^{2}\right)(\mathrm{n}=107)$ & 370.3 (range: 20.8-25111.5, IQR: 134.3-1034.2) \\
\hline
\end{tabular}

Abbreviations: ASO Amplatzer septal occluder device, IQR inter-quartile range, Qp:Qs ratio of systemic to pulmonary blood flow 
Table 3b

The use of multiple devices.

\begin{tabular}{|ll|}
\hline N & 21 \\
$\quad$ Two & \\
Three & $95 \%(20)$ \\
Multiple ASO* & $5 \%(1)$ \\
Helex and ASO & $76 \%(16)$ \\
Cribiform device and ASO & $10 \%(2)$ \\
\hline
\end{tabular}

* All were 2 ASO, except in 1 subject who received 3 ASO devices 


\section{Table 4}

Major and minor adverse events within 30 days of catheterization $(n=319)$

\begin{tabular}{|lc|}
\hline Event & Risk (95\% CI) \\
Major adverse event ${ }^{*}$ & $1.1 \%(0.4-2.6 \%)$ \\
Device embolization & $0.7 \%(0.1-2.0 \%)$ \\
Major device malposition & $0.4 \%(0.1-1.6 \%)$ \\
Minor adverse event & \\
Trivial residual shunt (1-3 mm) & $10.2 \%(6.7-14.7 \%)$ \\
$>3$ mm residual shunt & $0 \%(0-1.5 \%)$ \\
Minor device malposition & $0.9 \%(0.2-2.3 \%)$ \\
Trivial pericardial effusion & $5.7 \%(3.2-9.4 \%)$ \\
Hematoma & $6.0 \%(3.7-9.3 \%)$ \\
Transient atrioventricular block & $0.6 \%(0.1-2.2 \%)$ \\
Transient ectopic atrial tachycardia & $0.6 \%(0.1-2.2 \%)$ \\
Vascular injury & $0.6 \%(0.1-2.2 \%)$ \\
Headache & $4.3 \%(2.4 \%-7.1 \%)$ \\
\hline
\end{tabular}

Major adverse events were defined as those requiring repeat catheterization or other intervention, as opposed to minor adverse events, which did not require repeat catheterization or other intervention. 
Table 5

Multivariate analysis of candidate risk factors for composite outcome of technical failure or early major adverse outcome

\begin{tabular}{|lllll|}
\hline Risk factor & $\begin{array}{l}\text { Bi-variable screening } \\
\text { Odds Ratio (95\% CI) }\end{array}$ & $\boldsymbol{p}$ & $\begin{array}{l}\text { Multivariable Analysis } \\
\text { Odds Ratio (95\% CI) }\end{array}$ & $\boldsymbol{p}$ \\
\hline Deficient retro-aortic rim & $0.30(0.09-1.06)$ & 0.06 & $0.28(0.08-1.01)$ & 0.05 \\
Age at catheterization & $0.99(0.93-1.04)$ & 0.62 & & \\
Age < 3 years & $0.5(0.06-3.66)$ & 0.47 & & \\
Height & $1.01(0.99-1.02)$ & 0.48 & & \\
Weight & $1.00(0.99-1.02)$ & 0.61 & & \\
Qp:Qs & $2.0(1.1-3.4)$ & 0.02 & $2.57(1.22-5.40)$ & \\
Mean PA pressure & $1.06(0.96-1.2)$ & 0.27 & & \\
Defect Size & & & & \\
$\quad$ Frontal & $1.1(0.96-1.3)$ & 0.15 & & \\
Sagittal & $1.1(0.94-1.27)$ & 0.27 & & \\
Defect size on balloon sizing & $1.13(1.05-1.21)$ & 0.001 & & \\
Septal length & & & & \\
$\quad$ Frontal & $1.06(0.99-1.12)$ & 0.08 & & \\
Sagittal & $1.03(0.97-1.09)$ & 0.36 & & \\
Ratio of defect size to septal length & $0.6(0.00-660)$ & 0.9 & & \\
Defect size: BSA & $0.98(0.87-1.09)$ & 0.67 & & \\
Defect size: weight & $0.44(0.04-4.6)$ & 0.49 & \\
\hline
\end{tabular}

Abbreviations: BSA body surface area, CI confidence interval, Qp:Qs ratio of pulmonary to systemic blood flow

Point-estimates, confidence intervals, and $\mathrm{p}$-values for bi-variable screening are summarized. Those that met screening criteria $(\mathrm{p}<0.2)$ are italicized and were included in an initial multivariable model. Backwards selection was used to refine the model, based on stated criteria. The resultant final model is summarized in the columns for multivariable analysis. 


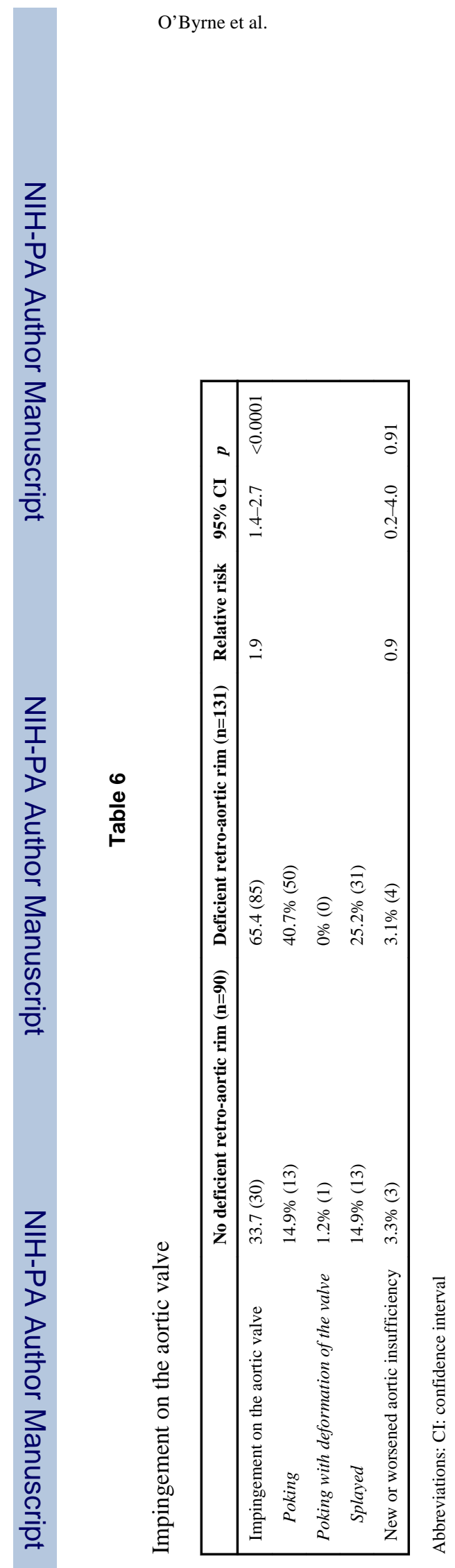

Page 20

Pediatr Cardiol. Author manuscript; available in PMC 2015 October 01. 